\title{
Nonperturbative Jackiw-Teitelboim gravity
}

\author{
Clifford V. Johnson \\ Department of Physics and Astronomy, University of Southern California, \\ Los Angeles, California 90089-0484, USA
}

(Received 12 March 2020; accepted 5 May 2020; published 26 May 2020)

\begin{abstract}
Recently, Saad et al. showed how to define the genus expansion of Jackiw-Teitelboim (JT) quantum gravity in terms of a double-scaled Hermitian matrix model. However, the model's nonperturbative sector has fatal instabilities at low energy that they be cured by procedures that render the physics nonunique. This might not be a desirable property for a system that is supposed to capture key features of quantum black holes. Presented here is a model with identical zperturbative physics at high energy that instead has a stable and unambiguous nonperturbative completion of the physics at low energy. An explicit examination of the full spectral density function shows how this is achieved. The new model, which is based on complex matrix models, also allows for the straightforward inclusion of spacetime features analogous to RamondRamond fluxes. Intriguingly, there is a deformation parameter that connects this nonperturbative formulation of JT gravity to one which, at low energy, has features of a super JT gravity.
\end{abstract}

DOI: 10.1103/PhysRevD.101.106023

\section{INTRODUCTION}

The Sachdev-Ye-Kitaev (SYK) model [1-3] has emerged as an important model of key dynamical phenomena in black hole physics. Of considerable interest is the thermal partition function $Z(\beta)=\exp \left(-\beta H_{\mathrm{SYK}}\right)$ and correlation functions thereof, which allow for the study of thermalization, quantum chaos, and other phenomena. The low energy sector of the physics has a dual description [4-7] in terms of Jackiw-Teitelboim (JT) gravity [8,9], a two-dimensional (2D) gravity theory whose partition function $Z(\beta)$ can be written (in a Euclidean presentation) as a topological expansion summing contributions from constant negative curvature surfaces of genus $g$ (the number of handles) with a boundary of fixed length $\beta$.

There is a Schwarzian action for the integral over the boundary. The $g=0$ (disc) contribution gives a result $Z_{0}(\beta)$ which can be interpreted [10], given the SYK connection, as

$$
Z_{0}(\beta)=e^{S_{0}} \int d E \rho_{0}(E) e^{-\beta E},
$$

where $\rho_{0}(E)$ is a spectral density function. Here, $S_{0}$ is a constant proportional to $1 / G$, where $G$ is the Newton constant of the 2D gravity. Correlation functions of powers

*johnson1@usc.edu

Published by the American Physical Society under the terms of the Creative Commons Attribution 4.0 International license. Further distribution of this work must maintain attribution to the author(s) and the published article's title, journal citation, and DOI. Funded by SCOAP. of $Z(\beta)$ can all be determined in terms of this spectral density function. The quantum chaotic dynamics of SYK have many features recognized [10-12] as suggestive of simpler models of large $N$ random matrix models, and indeed, in a recent beautiful paper by Saad et al. [13], the entire topological expansion for JT gravity was shown to be captured by a special type of model of random matrices-a Hermitian matrix model in a "double-scaling" limit in the sense defined in Refs. [14-17] in the context of defining a path integral over string world sheets. The double-scaled $1 / N$ expansion of the model, itself a genus expansion $[18,19]$, has its contributions at higher genus fully determined by a family of recursion relations [20-23] seeded by the spectral density $\rho_{0}(E)$, and this was shown [13] to be true for JT gravity also, with matching results, showing that the gravity theory is equivalent to a matrix model.

However, the matrix model has nonperturbative instabilities that show up at low energy. Their cure (outlined in Ref. [13]) renders the nonperturbative physics rather ambiguous. The purpose of this paper is to show how to construct a different matrix model definition that has the same perturbative physics as JT gravity at higher energy, but which possesses a well-defined nonperturbative sector, curing the physics at low energy. There are many attractive features of this new definition, and chief among them is the fact that the improved nonperturbative sector arises naturally in the matrix model, and furthermore that an underlying integrable structure (that also implicitly governs various topological recursion relations used in Ref. [13]'s perturbative work) suggests the nonperturbative completion.

It is worth borrowing a saying from a different context: while theoretical physics might not quite repeat itself, it 
often rhymes. The original double-scaled Hermitian matrix models of 1990 [14-17] yielded the first nonperturbative definitions of string theories. They were $2 \mathrm{D}$ gravity coupled to the $(2,2 k-1)$ conformal minimal models $(k=1,2, \ldots)$. It was swiftly recognized by the models' discoverers that the models with even $k$ had nonperturbative instabilitiesincluding the unitary "pure gravity" case of $k=2$. On the other hand, while not widely noticed, Refs. [24-28] showed that double-scaled complex matrix models can be defined that contain the same perturbation theory but better nonperturbative physics. The models (also indexed by $k$ ) also had a second perturbative regime with a distinct topological expansion that played a role in providing the good nonperturbative behavior, but was otherwise mysterious at the time. Much later, those models were interpreted in Ref. [29] as type 0A string theories: 2D gravity coupled to the $(2,4 k)$ superconformal minimal models.

As will be reviewed in the next section, there is a way of defining the JT gravity matrix model in terms of minimal models $[13,30]$, and so the lessons learned about how to define new minimal models with better nonperturbative physics can be used in the JT gravity context too. The JT gravity thus defined will have, in one regime, identical perturbation theory to the Saad et al. model, but far richer physics nonperturbatively. That nonperturbative physics will contain an interpolating pathway to the low energy physics of what can be recognized as a super JT gravity discussed recently in terms of matrix models by Stanford and Witten [31]. In some ways, the better nonperturbative behavior found here for the JT gravity definition can be attributed to the super JT gravity's (asymptotic) presence, repairing the low energy sector.

The paper is organized as follows: some key elements of the existing literature are briefly unpacked in Sec. II. In Sec. III, the focus is on the main workhorse of the matrix model, the (fully nonperturbative) spectral density. Clarity is maintained by first studying the simplest prototype case since it is enough to capture all the key features-the neighborhood of the tail end of the spectrum. After some review of this well-known "Airy" case in Sec. III A, a less familiar but relevant "Bessel" case is reviewed in Sec. III B. Next, the proposed spectral density with all the wellbehaved features it enjoys is explicitly constructed in Sec. III C. (For those who do not mind plot spoilers, it is displayed in Fig. 4 on page 10.) Additional aspects of the features of the spectral densities are studied in Sec. III D, where a differential equation that defines all the spectral densities discussed (and more besides) nonperturbatively is presented. Section III E uncovers some special features of the well-behaved spectral densities at $E=0$. There is an infinite family of distinct models possessing the good behavior revealed in Sec. III C, again indexed by integer $k$. In Sec. IV, it is shown how to combine them all in order to define a JT gravity matrix model with the desired stable, unambiguous nonperturbative behavior.
A closing discussion is presented in Sec. V, mostly outlining further steps for exploration of the many avenues this work seems to open up.

\section{THE SCHWARZIAN SPECTRAL DENSITY AND MINIMAL STRING MODELS}

Genus $g$ contributions in the gravity theory come with a weight factor $e^{S_{0}(1-2 g)}$, and so the matrix model topological expansion parameter is $\hbar=e^{-S_{0}}$. (This is the renormalized $1 / N$ after double-scaling and is thought of as a closed string coupling $g_{s}$ in the older 1990s context. The notation $\hbar$ will be used here in order to avoid confusion: the 2D gravity here is "spacetime," as opposed to world sheets for a string moving in some target spacetime derived from the "minimal matter" living on it.)

The particular disk partition function computed by the Schwarzian theory defines a spectral density [3],

$$
\rho_{0}(E)=\frac{1}{4 \pi^{2} \hbar} \sinh (2 \pi \sqrt{E}),
$$

and so this implicitly defines (perturbatively) the doublescaled matrix model to which the JT gravity is dual, since recursion relations yield the higher genus contributions to $\rho(E)$ in terms of it. One way to think about this definition is in terms of the infinite family of double-scaled matrix models describing gravity coupled to minimal models, labeled by an integer $k$, already mentioned in the Introduction. The $k$ th model has a spectral density at this order of the form [32] $\rho_{0}(E) \sim \sinh \left[(2 k-1) \cosh ^{-1}(1+E)\right]$. As was noted in Ref. [13], in the limit of large $k$, if $E$ is scaled as $1 / k^{2}$, this gives the Schwarzian spectral density in Eq. (2). So the JT gravity matrix model would appear to be a large $k$ limit of those minimal models. Alternatively, another connection was proposed in Ref. [30], and it will be returned to in Sec. IV. (However, the nonperturbative proposal presented here applies equally well to Ref. [13]'s way of connecting to the minimal models.) The partition function involves a trace of an effective one-dimensional Hamiltonian that arises naturally from the double-scaled matrix model [33] $\mathcal{H}=-\hbar^{2} \partial^{2} / \partial x^{2}+u(x)$, and so at leading order in $\hbar$,

$$
\begin{aligned}
Z_{0}(\beta, \mu) & =\int_{-\infty}^{\mu} d x\left\langle x\left|e^{-\beta \mathcal{H}(\hat{p}, \hat{x})}\right| x\right\rangle \\
& =\int_{-\infty}^{\mu} d x \int_{-\infty}^{+\infty} \frac{d p}{2 \pi \hbar} e^{-\beta\left[p^{2}+u_{0}(x)\right]} \\
& =\frac{1}{2 \hbar \sqrt{\pi \beta}} \int_{-\infty}^{\mu} d x e^{-\beta u_{0}(x)} \\
& =\frac{1}{2 \pi \hbar} \sqrt{\frac{\pi}{\beta}} \int_{u_{0}(\mu)}^{\infty} d u_{0} f\left(u_{0}\right) e^{-\beta u_{0}},
\end{aligned}
$$

where $u_{0}(x)=\lim _{\hbar \rightarrow 0} u(x)$ and $f\left(u_{0}\right)=-\partial x / \partial u_{0}$. (The normalization $\langle x \mid p\rangle=e^{i p x} / \sqrt{2 \pi \hbar}$ was used.) This can be written as 


$$
\begin{aligned}
Z_{0}(\beta, \mu) & =\int_{u_{0}(\mu)}^{\infty} d E \int_{u_{0}(\mu)}^{E} \frac{f\left(u_{0}\right)}{\sqrt{E-u_{0}}} \frac{d u_{0}}{2 \pi \hbar} e^{-\beta E} \\
& =\int_{u_{0}(\mu)}^{\infty} d E \rho_{0}(E, \mu) e^{-\beta E}
\end{aligned}
$$

where

$$
\rho_{0}(E, \mu)=\frac{1}{2 \pi \hbar} \int_{u_{0}(\mu)}^{E} \frac{f\left(u_{0}\right)}{\sqrt{E-u_{0}}} d u_{0} .
$$

So $\mu$ defines the end point of the classical distribution of energies. Taking $\mu \rightarrow 0$ (note that $u_{0}(0)=0$ ) yields Eq. (1), where (and henceforth) $\hbar^{-1}$ has been absorbed into the definition of $\rho_{0}(E)$. So $\rho_{0}(E)$ is determined if the leading piece of the potential of $\mathcal{H}$ is known. In fact, the general minimal model has this defining equation for $u_{0}$,

$$
\sum_{k=1}^{\infty} t_{k} u_{0}^{k}=-x,
$$

where $t_{k}$ is the coupling that turns on the $k$ th model. (Such an equation is the leading piece of what was called a "string equation" in the older matrix model literature.) If $t_{k}$ are specifically given by

$$
t_{k}=\frac{\pi^{2 k-2}}{2 k !(k-1) !},
$$

then $f\left(u_{0}\right)=I_{0}\left(2 \pi \sqrt{u_{0}}\right) / 2$, where $I_{0}(x)$ is the zeroth modified Bessel function. ${ }^{1}$ Putting this into Eq. (5) yields the Schwarzian spectral density given in Eq. (2). In this sense, the matrix model of JT gravity proposed in Ref. [13] is seen as being built by incorporating an infinite number of minimal models, each turned on just the right amount. Moreover, the construction above shows $[13,30]$ that the JT partition function is actually a "macroscopic loop" operator in the older minimal model language [33,34].

The next step is to consider the nonperturbative physics. The key elements are captured in the nonperturbative spectral density, discussed next.

\section{SPECTRAL CURVES AND NONPERTURBATIVE EFFECTS}

Potential problems with the nonperturbative physics emerge in the exact spectral density function, $\rho(E, \mu)$, the focus of this section. The simplest case (the leading behavior in small $E$ ) can be thought of as the $k=1$ model of the previous section (i.e., set $t_{1}=1$ and set all other $\left.t_{k}=0\right)$. Performing the integral (5) in this case yields the disc contribution $\rho_{0}(E, \mu)=(\pi \hbar)^{-1}(E+\mu)^{\frac{1}{2}}$. Higher

\footnotetext{
${ }^{1}$ Note that there is a normalization difference with Ref. [30], merely a matter of choice of conventions.
}

orders are yielded by recursion relations (see, e.g., Ref. [13]), but nonperturbative information must be sought elsewhere, in general cases. However, this prototype case can be solved exactly in terms of Airy functions, and it is reviewed in the next subsection. After that, an apparent digression will be presented in the subsection after detailing an analogous exact model involving Bessel functions. The next subsection describes the model of interest, which naturally combines features of both of these special cases. The two subsequent subsections present (respectively) a special differential equation that all the spectral densities solve (once the appropriate potential $u(x)$ is input) and a special analysis of the spectral density at $E=0$.

\section{A. The Airy case}

The prototype of the behavior of the Saad-ShenkerStanford [13] Hermitian matrix model of JT gravity can be seen in a special exact case that is built out of Airy functions. In fact, in the old language of the 1990s, it is the gravitating $(2,1)$ minimal model, $k=1$. It can be thought of as present in the very tip of the tail of all double-scaled Hermitian matrix models, and so in some sense is universal, as emphasized in this context in Ref. [13]. A most efficient way of discussing the matrix model is through the aforementioned effective Hamiltonian $\mathcal{H}$ that arises after doublescaling. It is

$$
\mathcal{H}=-\hbar^{2} \frac{\partial^{2}}{\partial x^{2}}+u(x)
$$

while $u(x)$ satisfies

$$
u(x)=-x .
$$

This is simply the $k=1$ specialization of Eq. (6). For this potential, the one-dimensional Schrödinger equation $\mathcal{H} \psi(E, x)=E \psi(E, x)$ is the defining equation for the Airy function $\mathrm{Ai}$ (up to rescalings to arrive at the conventional form), and the solution is

$$
\psi(E, x)=\hbar^{-2 / 3} \operatorname{Ai}\left(-\hbar^{-2 / 3}(E+x)\right) .
$$

It is useful for later (although trivial here) to keep in mind the physics represented by these wave functions. The potential is a straight line of unit negative slope, passing through zero at $x=0$. At a given $E$, far enough to the right there is oscillatory behavior. Moving to the left these oscillations eventually terminate at a peak which decays with an exponential tail as the wave function penetrates beyond the turning point $E=u=-x$. The translation invariance of the problem means that we have solutions with energies over the whole real $E$ line. 
The spectral density is defined in Ref. [13] to be

$$
\rho(E, \mu)=\int_{-\infty}^{\mu}|\psi(E, x)|^{2} d x
$$

although they have set $\mu=0$. Nonzero $\mu$ will be discussed here as well. The result can be deduced exactly, and one useful method for this [35] is to use the Airy kernel $K_{\mathrm{Ai}}(v, w)$ which is

$$
\int_{-\infty}^{0} \operatorname{Ai}(v+x) \operatorname{Ai}(w+x) d x=\frac{\operatorname{Ai}(v) \operatorname{Ai}^{\prime}(w)-\operatorname{Ai}(w) \operatorname{Ai}^{\prime}(v)}{v-w},
$$

where a prime denotes a derivative with respect to the argument. Here, $v$ and $w$ play the roles of energies and taking the limit where they are equal gives a finite result from the right-hand side (use L 'Hopital's rule). Using the defining equation for Airy to replace the second derivative gives the $\mu=0$ form in Ref. [13],

$$
\rho(E)=\hbar^{-\frac{2}{3}}\left[\operatorname{Ai}^{\prime}(\zeta)^{2}-\zeta \operatorname{Ai}(\zeta)^{2}\right],
$$

where $\zeta \equiv-\hbar^{-\frac{2}{3}} E$. The perturbative limit is at large $E$ (with wave functions that run to large negative $x$ ) and there $\rho(E) \rightarrow \rho_{0}(E)=(\pi \hbar)^{-1} \sqrt{E}$, the end point of the famous Wigner semicircle distribution. The nonperturbative corrections to this behavior are visible in two characteristic features: the first is that there are oscillations modulating the $\sqrt{E}$, becoming more pronounced at smaller $E$. These are earmarks of the underlying discreteness of the eigenvalues of the original matrix model, and the fact that they have a characteristic minimum spacing coming from their tendency to repel—an appealing feature of chaotic systems that also seem to be present in black hole physics, a motivating feature of this whole line of investigation $[2,3,10-12,36]$.

The second feature is the exponential tail of the distribution. In fact, it leaks into the $E<0$ region, referred to as the "forbidden region" in Ref. [13]. This feature is less desirable and in fact dangerous, since if the effective potential for an eigenvalue is negative in that regime, the system is unstable, with eigenvalues tunneling out of the distribution at $E \geq 0$ toward negative $E$. This is in fact what is observed in Ref. [13] for their matrix model of JT gravity. This is also consistent with the fact that for the $k$ even minimal models, the effective potential goes negative, making them nonperturbatively unstable. Since the JT gravity was shown in Sec. II to be built from an infinite number of such models, it inherits their affliction. Reference [13] offers options for curing this nonperturbative instability, but those are essentially user-defined choices - the physics is no longer unique. Below, a model will be presented which resembles the Airy case for large $E$,

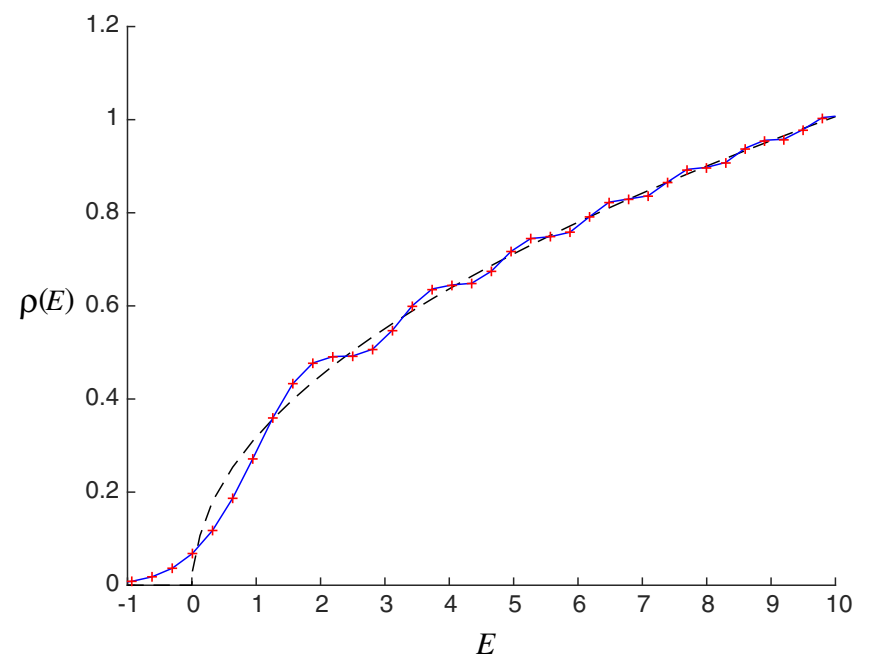

FIG. 1. The spectral density $\rho(E)$, both exactly (solid line) and numerically (crosses). The perturbative asymptote $\rho_{0}(E)=$ $(\pi \hbar)^{-1} \sqrt{E}$ is shown as a dashed line for comparison.

has the same kind of oscillatory modulations, but which avoids these problematic nonperturbative features.

In preparation for what is to come, it is worth treating this numerically, even though the exact answer is known. The Schrödinger problem was solved using a matrix version of the Numerov method [37], with $-100 \leq x \leq+100$ and a grid of $4000 \times 4000$ (it is easy to do larger grids but this turns out to be unnecessary). The 4000 eigensolutions thus found were suitably normalized ${ }^{2}$ and then the integral in Eq. (11) was performed (for $\mu=0$ ) using a simple trapezoidal routine. The result is displayed in Fig. 1, with cross marks representing the numerical points, and a continuous curve showing the analytical result of Eq. (13).

There is one final remark for this section, which will be important later. There is an additional parameter in the problem. The eigenvalue distribution end point can be placed somewhere other than $E=0$. It can be placed at $E=-\mu$ by integrating $x$ from $-\infty$ to $\mu$ in the defining integral for $\rho(E)$ in Eq. (11). Because of the translation invariance of the problem, it is a trivial shifting in the Airy kernel $K_{\mathrm{Ai}}(v, w)$ and simply replaces $E$ by $E+\mu$ in the result, giving

$$
\rho_{\mathrm{Ai}}(E, \mu)=\hbar^{-\frac{2}{3}}\left[\mathrm{Ai}^{\prime}(\zeta)^{2}-\zeta \operatorname{Ai}(\zeta)^{2}\right],
$$

with $\zeta \equiv-\hbar^{-\frac{2}{3}}(E+\mu)$, and the leading behavior $\rho_{\text {Ai }}(E, \mu)=(\pi \hbar)^{-1} E^{1 / 2}+\cdots$ emerges for $E \gg \mu$. Note

\footnotetext{
${ }^{2}$ There is a subtlety. Since the wave functions obtained, being free, are not square integrable, there is a normalization ambiguity that must be fixed. This can be done using the exact wave function solution in the Airy case of this section, and by judicious use of the analytically solvable behavior in certain asymptotic regimes in more nontrivial examples to come in later sections. These latter have been treated more carefully than in the first version of this paper, improving results for the small $E$ regime.
} 
that this shift does not help with the nonperturbative problems, ultimately, since the exponential leakage will always intrude into $E<0$. This is all trivial in this case, but will be useful for full appreciation of the results in subsequent subsections.

\section{B. The Bessel case}

Another illustrative exactly solvable case involves Bessel functions instead of Airy functions, and arises from having the potential

$$
u(x)=\frac{\hbar^{2}\left(\Gamma^{2}-\frac{1}{4}\right)}{x^{2}}
$$

in the Schrödinger problem above. Here $\Gamma$ is a constant. Its full significance will emerge later. The wave function $\psi(E, x)$ can again be solved exactly in terms of known functions, because [38] writing $\phi(x)=x^{-1 / 2} \psi(x)$ and rescaling $y=E^{1 / 2} x / \hbar$, the resulting equation is $y^{2} \phi^{\prime \prime}+y \phi^{\prime}+$ $\left(y^{2}-\Gamma^{2}\right) \phi=0$, which means that $\phi(y)=(\sqrt{2} \hbar)^{-1} J_{\Gamma}(y)$, a Bessel function of order $\Gamma$, where the prefactor is a convenient normalization. A spectral density for this problem can be solved for, using an analog of Eq. (11). This time, instead of a translation relation between $x$ and $E$, there is scaling one: rescaling $x$ to a value $\tilde{\mu}$ is equivalent to replacing $E^{\frac{1}{2}}$ by $E^{\frac{1}{2}} \tilde{\mu}$. So, defining $t=y^{2}$, the density can be written as

$$
\begin{aligned}
\rho_{J}(E, \tilde{\mu}) & =\int_{0}^{\tilde{\mu}}|\psi(E, x)|^{2} d x=\frac{1}{4 E} \int_{0}^{\frac{E \tilde{\mu}^{2}}{\hbar^{2}}} J_{\Gamma}^{2}(\sqrt{t}) d t \\
& =\frac{\tilde{\mu}^{2}}{4 \hbar^{2}}\left[J_{\Gamma}^{2}(\xi)+J_{\Gamma+1}^{2}(\xi)-\frac{2 \Gamma}{\xi} J_{\Gamma}(\xi) J_{\Gamma+1}(\xi)\right],
\end{aligned}
$$

where $\xi \equiv \tilde{\mu} \sqrt{E} / \hbar$.

Interestingly, in analogy with the Airy case, there is a kernel from which this can alternatively be derived $[39,40]$, the Bessel kernel $K_{J}(u, w)$,

$$
\begin{aligned}
\frac{1}{4} & \int_{0}^{1} J_{\Gamma}(\sqrt{v t}) J_{\Gamma}(\sqrt{w t}) d t \\
& =\frac{J_{\Gamma}(\sqrt{v}) \sqrt{w} J_{\Gamma}^{\prime}(\sqrt{w})-J_{\Gamma}(\sqrt{w}) \sqrt{v} J_{\Gamma}^{\prime}(\sqrt{v})}{\sqrt{v}-\sqrt{w}} .
\end{aligned}
$$

This Bessel case has leading large $E$ behavior,

$$
\rho_{J}(E, \tilde{\mu})=\frac{\tilde{\mu}}{2 \hbar \pi \sqrt{E}}-\frac{1}{4}\left(\Gamma^{2}-\frac{1}{4}\right) \frac{\hbar}{\tilde{\mu} \pi E^{3 / 2}}+\cdots,
$$

with oscillatory correction terms arising nonperturbatively, in analogy to the Airy case. A plot of the case $(\Gamma=0, \tilde{\mu}=$ $\sqrt{2})$ is given in Fig. 2. The classical singularity at $E=0$ is removed in the full behavior, and (for $\Gamma=0$ ) the density is a nonzero constant, $\tilde{\mu}^{2} / 4$, at $E=0$, the interpretation of which will be discussed further in the next subsection.

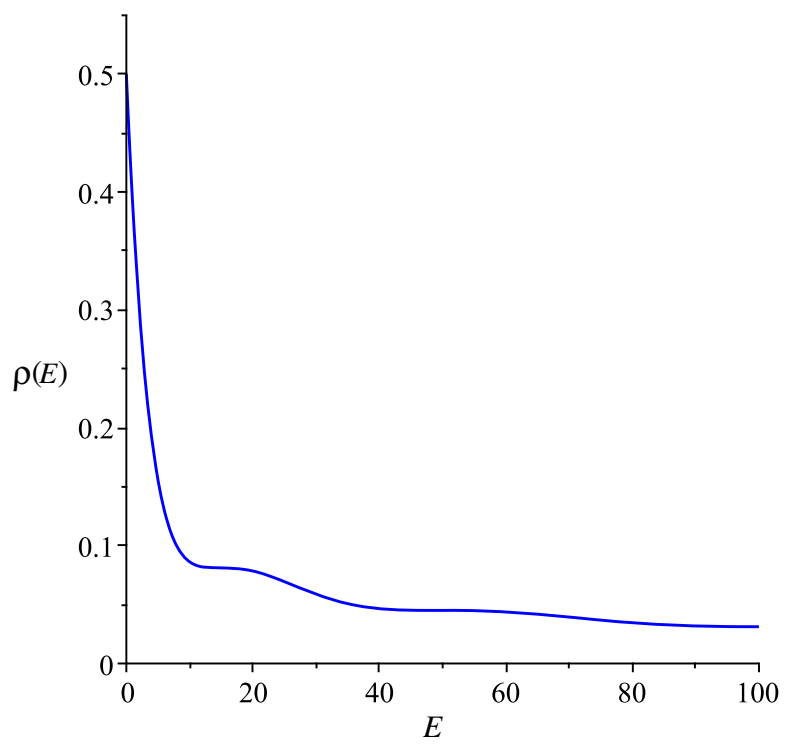

FIG. 2. The spectral density $\rho(E)$ for the Bessel case, with $\Gamma=0$ and $\tilde{\mu}=\sqrt{2}$.

Note that in the JT gravity context, Stanford and Witten [31] mentioned this case as part of a wider discussion generalizing the work of Ref. [13] to matrix ensembles other than the Hermitian case. In the same way that the pure Airy case serves as a prototype for the Saad-ShenkerStanford matrix model of JT gravity, this Bessel case should form the basis for a kind of super JT gravity. Indeed, they rightly point out that this Bessel behavior should arise as the tail of the spectral density in complex matrix models, since they fall into the $(\boldsymbol{\alpha}, \boldsymbol{\beta})=(1,2)$ Altland-Zirnbauer classification [41]. However, as will be made clear in the next subsection, it is only a special subsector of the physics that can be captured by complex matrix models, and it will be connected to much richer physics.

\section{Beyond Airy and Bessel}

Consider the following equation, which originally arose from double-scaling limits of complex matrix models $[24,26,27]$ :

$$
u \mathcal{R}^{2}-\frac{\hbar^{2}}{2} \mathcal{R} \mathcal{R}^{\prime \prime}+\frac{\hbar^{2}}{4}\left(\mathcal{R}^{\prime}\right)^{2}=0,
$$

where $\mathcal{R} \equiv u(z)+x$ and $u=u(x)$. A prime denotes an $x$ derivative. The Airy case of Sec. III A can also be thought of as a solution to this equation (i.e., $\mathcal{R}=0$ ). It turns out that the $\Gamma=0$ Bessel case of Sec. III B is also a special solution ${ }^{3}$ to this equation, the case of $\mathcal{R}=x$. This is the

\footnotetext{
${ }^{3}$ This curious exact solution was noticed in Ref. [42] and referred to as the $k=0$ solution. It was generalized to an interesting infinite family of rational solutions in Ref. [43] that were interpreted as string theories without D branes.
} 


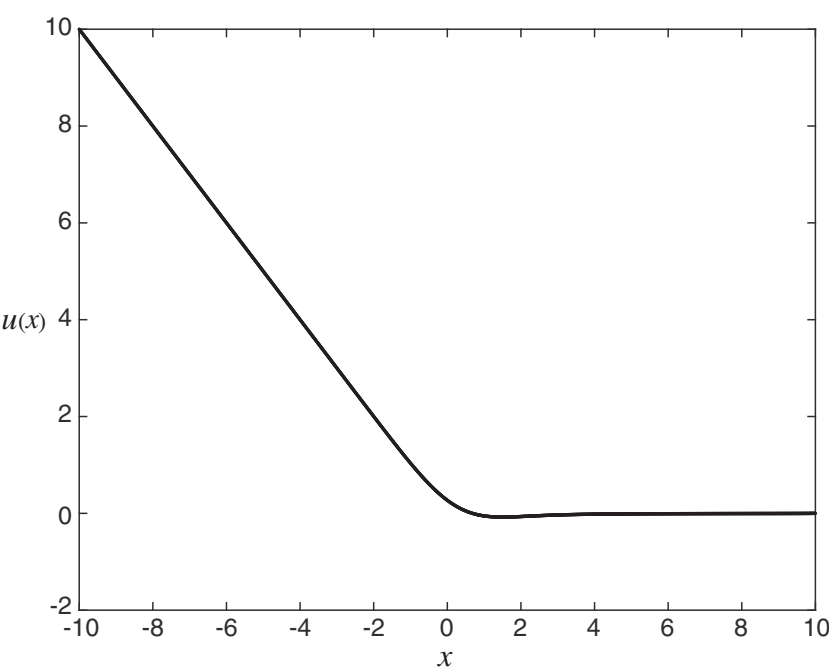

FIG. 3. A potential $u(x)$ that interpolates between the Airy case and the Bessel case. It is the unique $(k=1)$ solution to a special Eq. (19) derived from a matrix model.

realization of statements in Ref. [31] (see also Ref. [39]) that double-scaled complex matrix models can yield the Bessel-class tails for spectral densities. This behavior was already noticed in Refs. [24,26,27].

In this subsection however, a different solution to Eq. (19) will be used. For large negative $x$, the solution will behave as $u(x)=-x+O(\hbar)$, while for large positive $x$ it has $u(x)=0-\hbar^{2} / 4 x^{2}+O\left(\hbar^{3}\right)$. In fact, there is a unique solution and it is plotted (using MATLAB to construct it) in Fig. 3.

(For readers averse to numerics, uniqueness can in fact be proven analytically in this case [24]. Amusingly, the change of variables $u(x)=-x+2 y^{2}(x)$ reveals that $y(x)$ must solve the Painlevé II equation. For the asymptotics considered here, it has been shown [44] that there is a unique solution for $y(x)$.)

Crucially, the potential interpolates between those studied above for the Airy case and the Bessel case. There is a shallow well that connects them in the interior. In particular, for the one-dimensional Schrödinger problem, at high energies $E$, the physics will be similar to the Airy case. The low energy sector is different, however. Since the potential asymptotes to zero there is a state of energy $E=0$, the lowest, since the well turns out to support no bound states with $E<0[38,45]$.

The spectral density for this kind of model has not been studied nonperturbatively in the literature, but it is clearly important to examine it in the present context. Using the numerical techniques discussed in Sec. III A, the spectrum can be directly solved numerically and the spectral density function (11) explicitly constructed. The result for $\mu=0$ is given in Fig. 4, with the $\mu=0$ Airy case superimposed for comparison.

Several comments are worth making here. The first is that at large $E$ the spectral densities coincide. Furthermore,

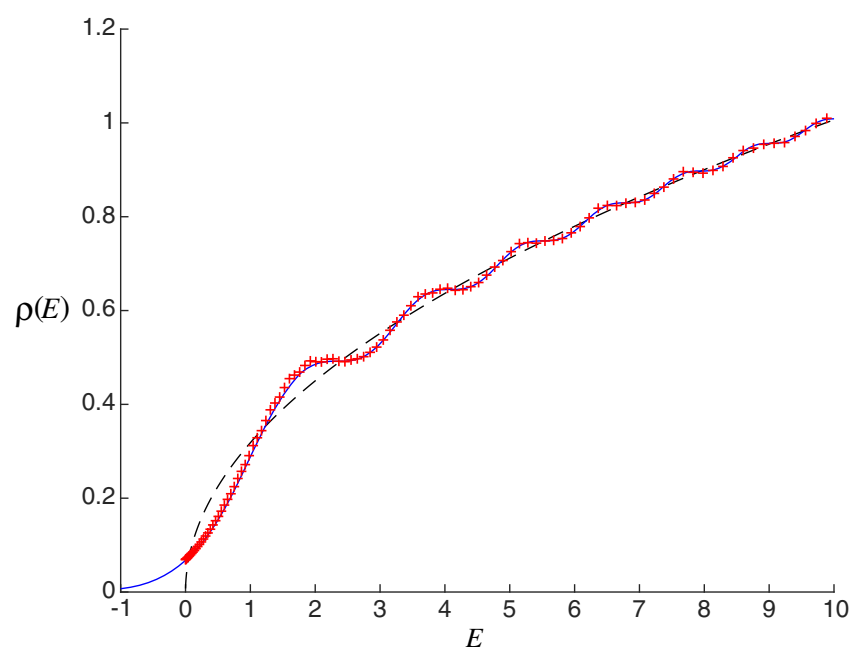

FIG. 4. The spectral density $\rho(E)$ extracted numerically (crosses) for the potential of Fig. 3, which is a solution of Eq. (19). For comparison, the exact Airy result is included (solid line). They differ significantly at low energies, with the new density terminating at a finite value at $E=0$. The perturbative asymptote $\rho_{0}(E)=(\pi \hbar)^{-1} \sqrt{E}$ is shown as a dashed line.

the oscillations that modulate the leading perturbative $\sqrt{E}$ behavior also match extremely well at high and intermediate energies, right down to deep into the low energy regime. There, the spectral density deviates from the Airy case, resulting in a slightly higher first peak, and approaches a nonzero constant at $E=0$, as will be confirmed in Sec. III E. ${ }^{4}$

Most crucially, there is no nonperturbative incursion into the forbidden region at all, for this model. In summary, this system, which is derived from a complex matrix model, has identical perturbation theory (large $E$ physics) to that presented in Ref. [13], shares many of the key nonperturbative features that are also desirable, but does not have the nonperturbative instability afflicting them at low $E$. Moreover, string equation (19) was shown in Refs. [26,27] to be the unique equation that follows from assuming only that the underlying Korteweg-de Vries (KdV) integrable structure of the minimal models be present nonperturbatively and a scaling symmetry. So, this lack of ambiguity in the nonperturbative completion should be inherited by the nonperturbative completion of JT gravity proposed here.

There is more, however. Turning on the parameter $\mu$ here is less trivial than before, because the potential $u(x)$ is not translationally invariant. For either sign of $\mu$, at large $E$ the density function asymptotes to $(\pi \hbar)^{-1} \sqrt{E}$, as before. For increasingly negative $\mu$, the $E=0$ value of the density

\footnotetext{
${ }^{4}$ Determining precisely whether the density drops sharply to zero precisely at $E=0$, or reaches a constant there, is hard to confirm using this approach, since there is no direct control over which eigenstates are sampled. A different method for $E=0$ will be employed in Sec. III E, confirming that $\rho \neq 0$ and $E=0$.
} 


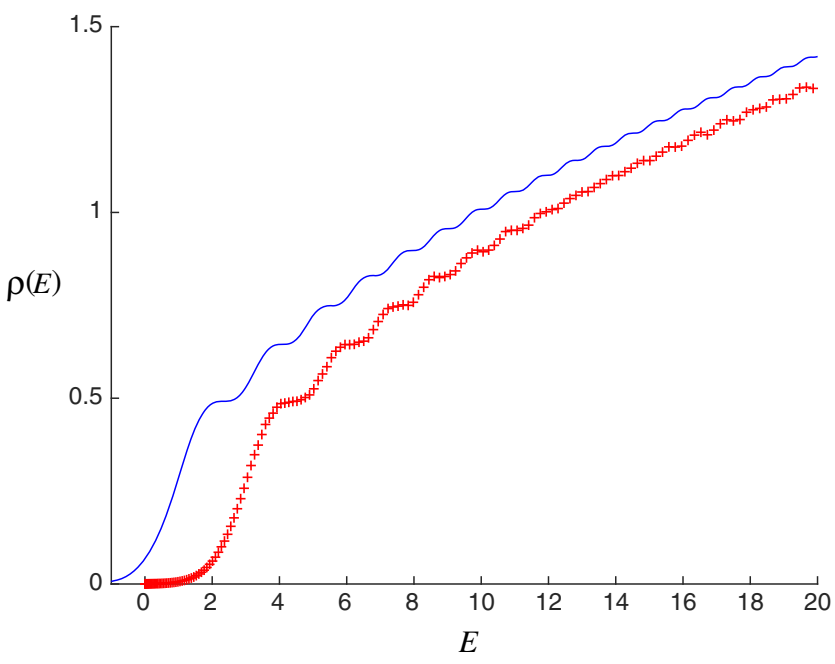

FIG. 5. The spectral density $\rho(E)$ extracted numerically (crosses) for the potential of Fig. 3, which is a solution of Eq. (19). For comparison, the exact Airy result is included (solid line). Parameter $\mu=-2$ has been turned on, pushing the distribution to the right.

approaches zero (see Sec. III E), and now the bulk of the distribution is pushed to the right, as shown in Fig. 5. In fact, for negative enough $\mu$, the density looks increasingly like the (translated) Airy case (except that the tail terminates at $E=0$ ). For positive $\mu$ however, the behavior is strikingly different, as shown in Fig. 6.

The distribution moves to the left, increasing its value in the neighborhood of $E=0$, looking increasingly there like the Bessel case studied in the previous section: for increasingly large positive $\mu$, the density $\rho(E)$ dips sharply from an increasingly high value, before eventually merging into the undulations of the Airy-like sector.

At finite $\mu$, it cannot ever resemble the Bessel case exactly, since there are hybrid Airy-Bessel-like wave functions present. Tuning to larger $\mu$ probes more of the regime with the Bessel-relevant potential (15), with more participation of the spectral density $\rho_{J}(E, \tilde{\mu})$ of Eq. (16) with a $\tilde{\mu}$ that grows with $\mu$. So the limit $\mu \rightarrow \infty$ combined with an infinite rescaling down of the vertical axis would yield the pure Bessel case, giving the finite (after rescaling) $\rho_{J}$ at $E=0$ seen for Bessel. Meanwhile, the rescaling also flattens away the features due to Airy into the horizontal axis, resulting in Fig. 2.

This special limit (once it is built into a complete model in Sec. IV) makes contact with the aforementioned super JT gravity model recently discussed by Stanford and Witten [31] in this context. The finite $\mu$ physics retains access to the perturbative regime that matches what is needed for ordinary JT gravity at high energy, but connects it to better nonperturbative physics that does not include incursions to $E<0$, a desirable feature for the goal of this paper.

The interpretation of all this behavior with $\mu$ is that there is a natural infinite "wall" at $E=0$ in this matrix model, past

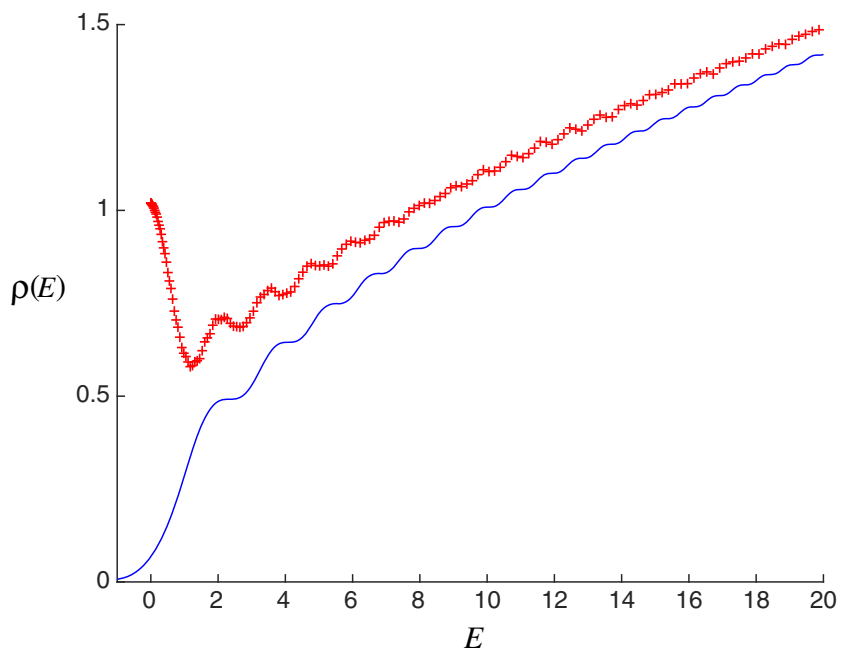

FIG. 6. The spectral density $\rho(E)$ extracted numerically (crosses) for the potential of Fig. 3, which is a solution of Eq. (19). For comparison, the exact Airy result is included (solid line). Parameter $\mu=2$ has been turned on, pushing the distribution to the left, where the eigenvalues "pile up" at $E=0$.

which eigenvalues cannot flow. ${ }^{5}$ This is in sharp contrast to the standard Hermitian case used for the definition of Ref. [13]. The position of the eigenvalue distribution's end point is controlled by $\mu$. In the present construction, negative values of $\mu$ move it away from the wall, while positive values push it into the wall. There, the eigenvalues pile up against the wall since they cannot go to $E<0$. (The Bessel system of the previous section is an exact model of this latter phenomenon.) While it can be set to zero, since $\mu$ is clearly a meaningful nonperturbative parameter in this model, it will be kept and interpreted in later sections.

Of course, the Airy case of Sec. III A is just a model of the full JT gravity matrix integral's features, ${ }^{6}$ but it did indeed capture key essences. Similarly, the model presented in this section exhibits key aspects of a matrix definition that has rather attractive nonperturbative features while retaining all the perturbation theory of the Airy case. The job of Sec. IV is to show how this is incorporated into a fully operational new nonperturbatively improved model of JT gravity. Before doing that, it is worth making an observation about an exact differential equation (in fact a whole family of them) obeyed by the spectral densities discussed so far.

\section{A differential equation for spectral densities}

It is possible to derive a special differential equation for each of the spectral densities discussed, which has a

\footnotetext{
${ }^{5}$ In the original complex matrix model context in which some of these features were discovered, the wall $[24,26]$ can be traced to the fact that integrating out the angular variables left the eigenvalues defined on the real positive line.

${ }^{6}$ In the language of Steve Shenker's Strings 2019 talk, it is a model of a model of a model...of a model.
} 
universal form that may make it useful for further studies of models of JT gravity. In a sense, it may be thought of as a complementary tool to the loop equation or recursion approach of Refs. [13,31]. The effective Hamiltonian, $\mathcal{H}$, that emerges in the double-scaling limit [see Eq. (8)] has a resolvent associated to it: $\hat{R}(x, E) \equiv\left\langle x\left|(\mathcal{H}-E)^{-1}\right| x\right\rangle$. It actually satisfies the Gel'fand-Dikii equation [46],

$$
4(u-E) \hat{R}^{2}-2 \hbar^{2} \hat{R} \hat{R}^{\prime \prime}+\hbar^{2}\left(\hat{R}^{\prime}\right)^{2}=1,
$$

where $u=u(x)$, and a prime denotes a differentiation with respect to $x$. Crucially, this is not the matrix model resolvent that is discussed in Refs. [13,31], although they are related. The latter is obtained from the former, $\hat{R}(x, E)$, by integrating once with respect to $x$, and then evaluating it at $\mu=0$. (As stated before, more physics can be seen by retaining the $x$ dependence, as is done here.) The object obtained by integrating once is, in the old matrix model language, the Laplace transform of the (double-scaled) loop operator expectation value, denoted $w(E, x)$ here [and not $R(x, E)]$ to avoid notational confusion. Its imaginary part (divided by $\pi$ ) yields the double-scaled spectral density. ${ }^{7}$

Given this connection, a differential equation for $\rho(E, \mu)$ can be derived directly by writing $\rho^{\prime} \sim \operatorname{Im}(\hat{R})$, substituting into Eq. (20), yielding a third order equation,

$$
4(u-E)\left(\rho^{\prime}\right)^{2}-2 \hbar^{2} \rho^{\prime} \rho^{\prime \prime \prime}+\hbar^{2}\left(\rho^{\prime \prime}\right)^{2}=1 .
$$

This is highly nonlinear, but a simpler equation can be derived by taking an extra derivative. A derivative of Gel'fand-Dikii results in an overall factor of $\hat{R}$ that can be divided out, and so after substituting

$$
\hbar^{2} \rho^{\prime \prime \prime \prime}=2 u^{\prime} \rho^{\prime}+4(u-E) \rho^{\prime \prime},
$$

which, for a given potential $u(x)$, defines $\rho(E, x)$ to all orders in perturbation theory and beyond, after specification of the appropriate choice of boundary conditions. Setting $x=\mu$ yields the desired $\rho(E, \mu)$. Again, the facility of having the variable $x$ (and hence $\mu$ ) manifest is apparent here.

This differential equation [in either form (21) or (22)] is a remarkably compact and universal form as a nonperturbative definition of the spectral densities. The main input is the form of $u(x)$, which is determined by which of a number of types of matrix model is being discussed. It is instructive to check that the exact Airy and Bessel spectral densities $\rho_{\mathrm{Ai}}(E, \mu)$ and $\rho_{J}(E, \mu)$ [given in Eqs. (14) and (16)] derived in the previous sections for the potentials

\footnotetext{
${ }^{7}$ So the Laplace transform is $w(\ell, x)$, the expectation value of loops of length $\ell$. In the present context, $\ell=\beta$, the inverse temperature of the SYK model, and the loop expectation value is essentially the SYK/JT partition function.
}

$u(x)=-x$ and $u(x)=\hbar^{2}\left(\Gamma^{2}-\frac{1}{4}\right) / x^{2}$, respectively, do indeed satisfy the equation (in either form).

This also gives an alternative way of solving numerically for the nonperturbatively desirable spectral density of the previous section, with that interpolating $u(x)$ (which solves Eq. (19), derived from complex matrix models) as input. In fact, solving it at or near $E=0$ could give an alternative way of getting better numerical resolution in that regime. Unfortunately, the differential equation (in either form) is extremely sensitive to numerical instabilities in precisely this regime, and so no insights into $E=0$ were gained here. The equation certainly deserves further study however, and moreover it will be helpful in precisely phrasing a nonperturbative formulation of JT gravity in Sec. IV. As for better understanding of $E=0$, a different approach was taken and is described next.

\section{E. The spectral density at $E=0$ and the Miura transformation}

Section III C uncovered the properties of the spectral density with the desirable nonperturbative properties, but the methods used were not well adapted to deliberate and precise exploration of the neighborhood of $E=0$. Going to higher and higher resolution in the discretized scheme yields eigenvalues of successively lower energies, but this only allows an approach to $E=0$ asymptotically. This made it hard to cleanly determine whether the density $\rho(E, \mu)$ approached a finite value there, or whether it drops precipitously to zero. The differential equation for the density of the previous section contains the answers, in principle, but is hard to control numerically at low energies. There is another approach, however.

As pointed out in Ref. [38] for just this kind of system, $E=0$ is rather special in that the wave function can be succinctly characterized in terms of a differential equation: factorizing according to $\mathcal{H} \equiv(-\hbar \partial+v)(\hbar \partial+v)$, where $u=v^{2}-\hbar v^{\prime}$, the wave function $\psi(E=0, x)$ is annihilated by $(\hbar \partial+v)$, and hence

$$
\psi(E=0, x)=A \exp \left\{-\hbar^{-1} \int^{x} v\left(x^{\prime}\right) d x^{\prime}\right\}
$$

where $A$ is a normalization constant. (Factoring in the other order simply changes the sign on $v$.) In fact, the relation $u=v^{2}-\hbar v^{\prime}$ defines the well-known (in the classic integrable systems literature) "Miura transformation" or "Riccati relation" between the KdV and mKdV integrable systems. More specifically though, it was shown in Ref. [42] that when $u(x)$ satisfies the defining Eq. (19), with the desired boundary conditions $u=-x+\cdots$ $(x \rightarrow-\infty)$ and $u=0+\cdots(x \rightarrow+\infty)$, the function $v(x)$ actually satisfies the following differential equation: 


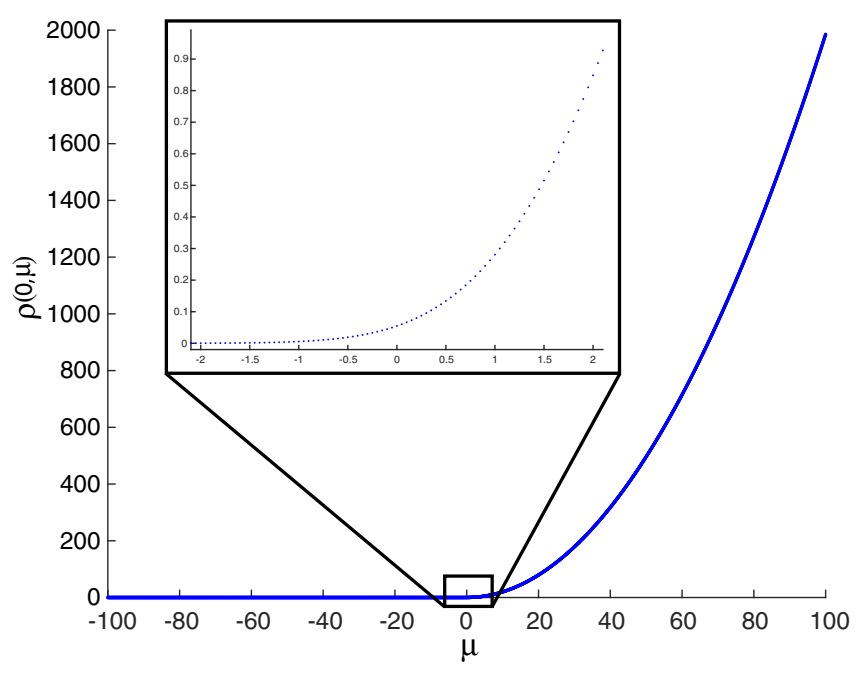

FIG. 7. The spectral density at zero energy, $\rho(0, \mu)$. The inset enlarges the behavior near $\mu=0$.

$$
\frac{\hbar^{2}}{2} v^{\prime \prime}-v^{3}-x v+\frac{\hbar}{2}=0
$$

with $v=(-x)^{\frac{1}{2}}-\hbar / 4 x+\cdots(x \rightarrow-\infty)$ and $v=0+\cdots$ $(x \rightarrow+\infty)$. In fact, Eq. (24) is a celebrity, the Painlevé II equation, with a specific value for its constant. ${ }^{8}$ It is a rather simple and well-behaved differential equation to tackle numerically, and $v(x)$ can readily be found, along with its first integral (MATLAB was used). Hence, the spectral density at zero energy was computed,

$$
\rho(E=0, \mu)=A^{2} \int_{-\infty}^{\mu} d x|\psi(E=0, x)|^{2},
$$

where $A$ is yet to be determined. A plot of this density (for $A=1$ ) is shown in Fig. 7, and it is in accord with expectations: for very negative $\mu$, the integration over $x$ from $-\infty$ to $\mu$ to make the density $\rho(E, \mu)$ picks up mostly contributions from high energy wave functions. There is very little contribution from low energy states, since those are mostly localized to the right (recall the form of the potential $u(x)$ in Fig. 3), with only small exponential tails penetrating to the left. For positive $\mu$, the zero energy sector can contribute strongly, since the integral now covers the region where it is most supported. From this perspective, it is not surprising, therefore, that $\rho(E=0) \neq 0$ at $\mu=0$, receiving a contribution from the tail of the $E=0$ wave

\footnotetext{
${ }^{8}$ This is a different appearance of Painlevé II than was mentioned in passing two paragraphs below Eq. (19). Fans of Painlevé transcendents should note that Painlevé I appeared as the string equation for the original (nonperturbatively unstable) double-scaled Hermitian matrix model [14-17], and more recently Painlevé IV made an appearance as a string equation arising from a reduction of the "dispersive water wave hierarchy" $[47,48]$.
}

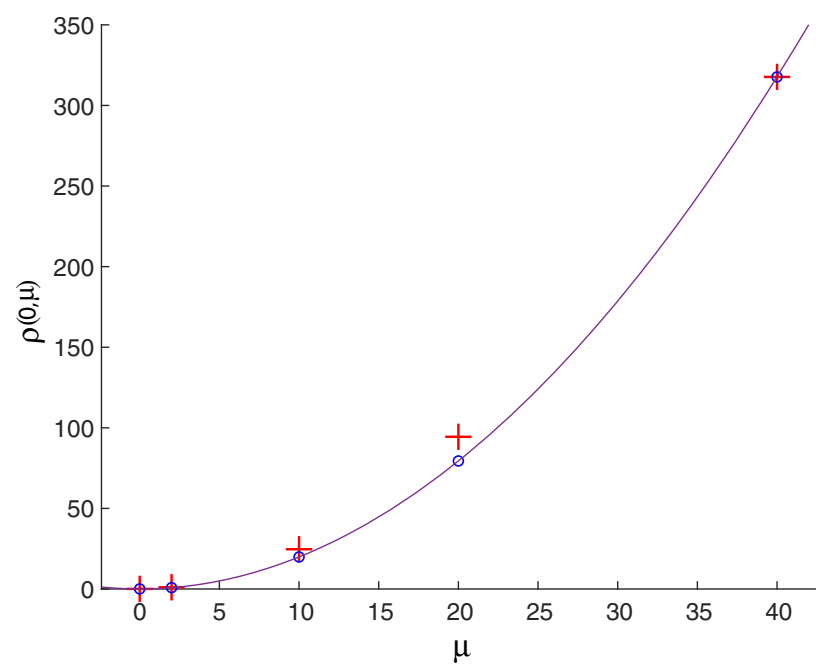

FIG. 8. Comparison of choice values of the spectral density at/ near zero energy, $\rho(0, \mu)$ computed by two separate methods. See text for details.

function, as can be seen in the figure. This confirms the numerical suggestions about this regime, done in Sec. III C by sampling the spectrum.

In fact, this result can be used as further independent confirmation of the methods of Sec. III C since the value that the density approaches as $E=0$ is approached should have the same $\mu$ dependence as seen in the curve of Fig. 7 , up to an overall scale since $A$ (above) was unfixed. A successful check was done, using a sample of five points at $\mu=0,2,10,20,40$, as shown in Fig. 8, where comparing two points fixed $A^{2} \simeq 39.68$.

In the figure, the circles are the values of $\rho(E=0, \mu)$ as computed in Eq. (25) using the methods of this subsection (in this range of $\mu$ values the increase with $\mu$ is actually quadratic, to good accuracy) while the "experimental" data marked by the crosses are the nonzero values read off for $\rho(E=0, \mu)$ at the closest approach to $E=0$ available for the discrete spectrum-sampling system used in Sec. III C. (That lowest value was $E \simeq 5.8 \times 10^{-4}$.)

\section{NONPERTURBATIVE JT GRAVITY DEFINED}

There is an infinite family of models with the same character as the one discussed in Sec. III C, originally defined using complex matrix models and studied extensively in Refs. [24-28,49]. They are indexed by an integer $k$ (Sec. III C example is $k=1$ ) and were later identified [29] in the string theory context as nonperturbative definitions of the $(2,4 k)$ superconformal minimal string models. The function $u(z)$ that the matrix model defines in the doublescaling limit is a solution of the string equation (19) with

$$
\mathcal{R} \equiv \tilde{R}_{k}[u]+x
$$

where $\tilde{R}_{k}[u]$ is the $k$ th polynomial in $u(x)$ and its $x$ derivatives defined by Gel'fand and Dikii [46], but 


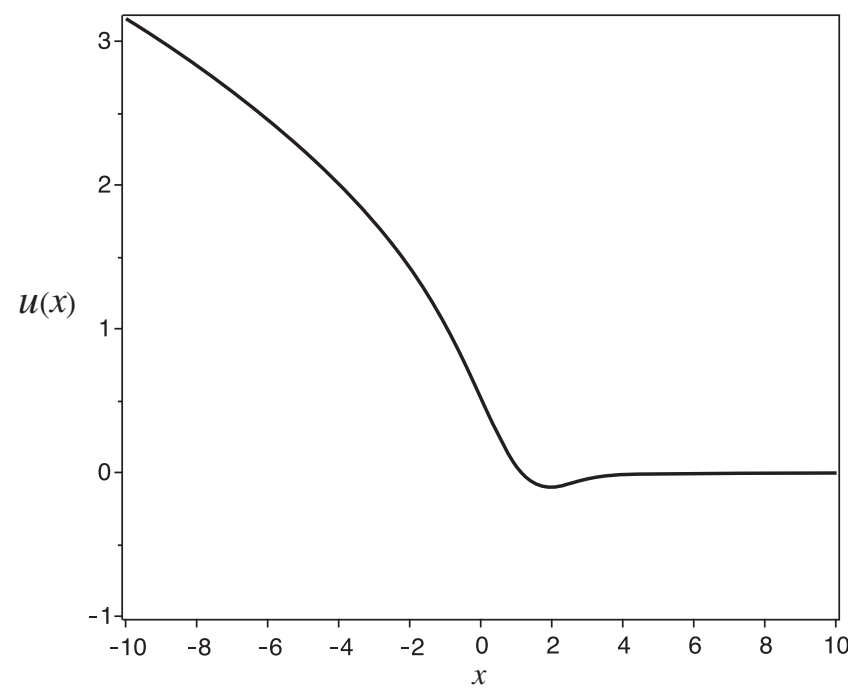

FIG. 9. The potential $u(x)$ that is supplied by Eq. (19) for the case $k=2$, where asymptotically $u(x)=(-x)^{1 / 2}+\cdots$ to the left and $u(x)=-\hbar^{2} / 4 x^{2}+\cdots$ to the right.

normalized so that the coefficient of $u^{k}$ is unity. The original $(2,2 k-1)$ bosonic minimal models are equivalent to taking the $\mathcal{R}=0$ solution. Instead, the models of interest have $u(x)=(-x)^{1 / k}+\cdots$ for negative $x$ and $u(x)=$ $-\hbar^{2} / 4 x^{2}+\cdots$ for positive $x$. (Note that the leading positive $x$ behavior is $k$ independent, showing a kind of universality.) They have the same perturbative expansion in negative $x$ as the gravitating $(2,2 k-1)$ minimal models, but better nonperturbative behavior due to their distinct positive $x$ behavior. Aspects of the physics of these models have been studied a great deal. (While the leading perturbative behavior of the spectral density was studied in both the positive and negative $x$ regimes long ago in Refs. [26,27], characterizing the effects of the wall, the detailed computation and analysis of the nonperturbative form of the spectral density presented here is new, however, as is their definition via a differential equation, given in Sec. III D.)

For the Schrödinger problem of Eq. (8), with the potential $u(x)$ possessing the asymptotics described, there is again a well in the intermediate region, and studies suggest ${ }^{9}$ that it is too shallow to support bound states. This means that all of these models have well-behaved stable nonperturbative physics, and their low energy behaviorsthe very tail of the spectral density - are all controlled by the features exhibited in the previous subsections. (For the $E=0$ analysis of the subsection immediately preceding, the generalization for higher $k$ involves writing a wave function again of the form of Eq. (23), with $v(x)$ solving a higher $k$ generalization of Painlevè II. See Refs. [38,42].)

\footnotetext{
${ }^{9}$ It has not been proven, but numerical and analytical work $[38,45]$, along with the ability to diagonalize the parent complex matrix model into positive eigenvalues, suggest that it is true.
}

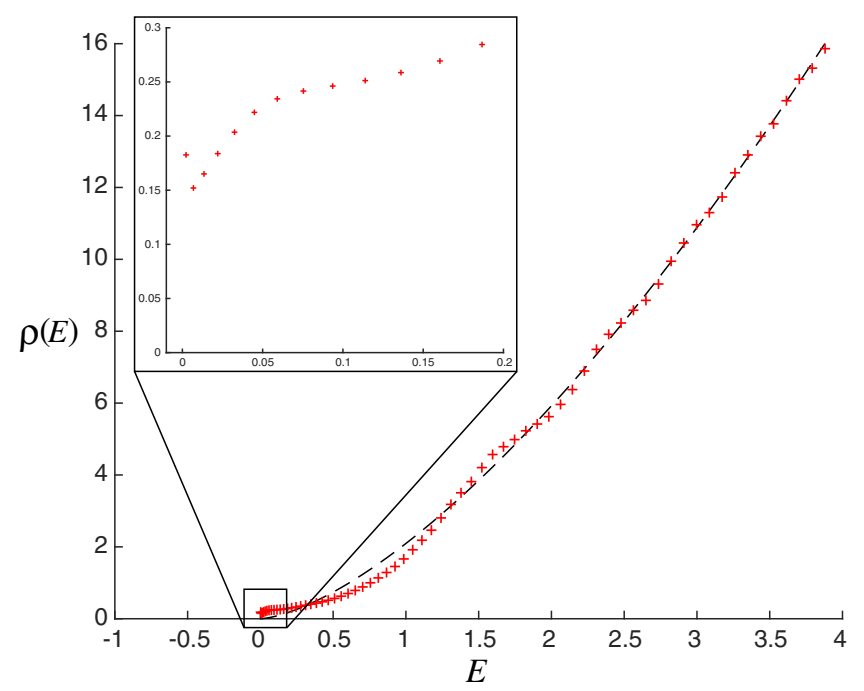

FIG. 10. The spectral density $\rho(E)$ extracted numerically (crosses) for the $k=2$ potential of Fig. 9, which is a solution of Eq. (19). It approaches a constant value at $E=0$ (see inset: it is not clear if the upward displacement of the leftmost point is a numerical artifact or not, but it does not change the conclusion). The perturbative asymptote $\rho_{0}(E)=2 \pi E^{3 / 2} / 3 \hbar$ is shown as a dashed line.

As an additional example, the $k=2$ case was solved numerically and displayed in Fig. 9. The spectral density was computed using the same numerical techniques described in Sec. III C, and is displayed in Fig. 10, for $\mu=0$. This density asymptotes to $\rho_{0} \sim E^{3 / 2}$ (the known perturbative result shared also by Hermitian matrix models: $\rho_{0} \sim E^{k-\frac{1}{2}}$ for the $k$ th model) and again approaches a small nonzero value at $E=0$, as shown in the inset.

It is clear how to define the full nonperturbatively well-defined matrix model for JT gravity that was promised. The general interpolating model defines a potential $u(x)$ as a solution to the string equation (19) with $\mathcal{R} \equiv$ $\sum_{k} t_{k} \tilde{R}_{k}[u]+x$. Turning on the same combination of an infinite number of $t_{k} \mathrm{~s}$ as defined in Eq. (7) will ensure that the disc partition function will define the same leading spectral density at large $E$ displayed in Eq. (2). (As mentioned previously, this is because the large $-x$ regime for Eq. (19) is perturbatively the same as solving $\mathcal{R}=0$, the Hermitian matrix model string equation.) As seen, at any order in perturbation theory $(E \gg \mu)$, the physics will be the same, but as $E \sim \mu$ or lower, the physics will be different, and of the character shown in Figs. 4, 5, 6, and 10. The full spectral density is supplied by the differential equation in the form (21) or (22). As an alternative, the large $k$ limit suggested in Ref. [13] (see also Ref. [31]) could also work and should be explored further.

\section{DISCUSSION}

The core result of this work is a construction of a matrix model of JT gravity that exhibits the same physics at high $E$ 
as the matrix model of Saad, Shenker, and Stanford (SSS) [13], matching on to perturbative JT gravity, but that has a better nonperturbative sector in that it is stable and unambiguous. How the low $E$ physics differs from the SSS model depends upon a parameter $\mu$. [It is normally set to zero (in the approach of SSS), but it is natural to explore different values of it to better understand the physics.] This construction was achieved by building it out of an infinite family of special minimal models, in the same way that the SSS definition can be built $[13,30]$ out of an infinite family of minimal models derived from double-scaled Hermitian matrix models. The special minimal models used for the new construction were derived long ago using doublescaled complex matrix models, with the key physics being output in the form of the string equation (19) studied extensively by Dalley, Johnson, and Morris (DJM) [26-28]. For any of the individual models, the equation's solution defines a fully nonperturbative potential $u(x)$ for the Hamiltonian $\mathcal{H}=-\hbar^{2} \partial^{2} / \partial x^{2}+u(x)$ from which a nonperturbative spectral density $\rho(E)$ can be extracted, as was done explicitly here for the first time. It enjoys (as demonstrated explicitly in Sec. IIIC for the prototype $k=1$ case and in Sec. IV for the case of $k=2$ ) the advertised nonperturbative features, having no incursion into the forbidden region $E<0$, in contrast to the Airy-like behavior that is the foundation of the SSS model. Matrix eigenvalues do not tunnel to oblivion in this class of models. (See Figs. 1 and 4 for the comparison.)

The construction yielded some fascinating bonus features. The nontrivial parameter, $\mu$, deforms the theory continuously toward the physics of a type of super JT gravity discussed recently by Stanford and Witten [31]. This intriguing connection deserves to be better understood in its own right. It could also give insights into SYK-type models, and ultimately into phases of black hole physics, given the interconnectedness of all these systems. There is a constant, $\Gamma$, that can naturally be present in the model. It was switched off for most of this paper, but can easily be incorporated into the string equation (19),

$$
u \mathcal{R}^{2}-\frac{\hbar^{2}}{2} \mathcal{R} \mathcal{R}^{\prime \prime}+\frac{\hbar^{2}}{4}\left(\mathcal{R}^{\prime}\right)^{2}=\hbar^{2} \Gamma^{2},
$$

where $\Gamma$ was recognized [42] as introducing open string world sheets into the topological expansion.

In fact, $\Gamma$ counts background $D$ branes and R-R fluxes in the type 0A minimal model interpretation of Ref. [29]. Its role in that context was further elucidated and explored in Refs. $[38,45,50]$. Here in this JT gravity context it should be expected to be associated with additional spacetime boundaries and Ramond insertions.

This generalization to include $\Gamma$ is what introduces the $\Gamma^{2}$ term in Eq. (15), resulting in Bessel functions of order $\Gamma$. In fact, $\Gamma$ would seem to be identified with the parameter $\nu$ in
Sec. 5.5 of the paper of Stanford and Witten [31]. So the system with $\Gamma$ turned on is in an ensemble of the $(\boldsymbol{\alpha}, \boldsymbol{\beta})=$ $(1+2 \Gamma, 2)$ type in the Altland-Zirnbauer classification [41]. Moreover, the observations in Ref. [38] that $\Gamma$ counts the number of threshold bound states in a supersymmetric quantum mechanics problem is connected to similar observations made in Ref. [31] about super JT gravity. The cases $\Gamma= \pm \frac{1}{2}$, which give the special $(\boldsymbol{\alpha}, \boldsymbol{\beta})=$ $(\{0,2\}, 2)$ cases discussed there, correspond nicely to the vanishing of the entire topological expansion in the positive $x$ regime of the string equation. This will be explored further [51].

In fact, it was discovered a while ago in Ref. [43] that for half-integer $\Gamma$ there are special solutions of the string equation (27) that are rational functions for any $k$, generalizing the simple Bessel case. They were studied there as peculiar examples of string theories that had no D-brane sectors. Various quantities such as the resolvent and the spectral densities have simple exact expansions. It is natural to suppose that these solutions might be useful for understanding further features of (possibly new types of) super JT gravity.

It is worth noting that the DJM string equation (27) can actually be derived without appealing to any particular matrix model, but instead assuming that the underlying integrable structure - the KdV hierarchy in this case-persists at the nonperturbative level. A scaling argument combined with the recursion relation for the Gel'fand-Dikii polynomials yields a total derivative of the equation. Integrating once and setting the constant to $\hbar^{2} \Gamma^{2}$ yield the result $[27,42]$.

In the mathematical literature, an ordinary differential equation obtained in this way is described as arising from a similarity reduction of an integrable partial differential equation (PDE). In fact, the type OB minimal models obtained from double-scaling unitary matrix models (or multicut Hermitian ones) have string equations in this class, the integrable system being the Zakharov-Shabat hierarchy [29,52-55]. This suggests that the structures found so far are a tip of a large iceberg. In fact, it was conjectured [47] that for the right classes of integrable families of PDEs, such similarity reductions might always yield string theories, without the need to derive them from double-scaling limits of matrix models. Explorations along those lines produced several interesting results and uncovered new stringlike theories $[47,48]$ by starting from the dispersive water wave hierarchy. Perhaps in the spirit of what was done in this paper, some of these classes of models (or other models to be defined using that scheme) could be combined to yield new types of 2D gravity of the JT type.

\section{ACKNOWLEDGMENTS}

C. V.J. thanks the U.S. Department of Energy for support under Grant No. DE-SC 0011687 and Amelia for her support and patience. 
[1] S. Sachdev and J. Ye, Phys. Rev. Lett. 70, 3339 (1993).

[2] A. Kitaev, KITP Seminars (2015).

[3] J. Maldacena and D. Stanford, Phys. Rev. D 94, 106002 (2016).

[4] A. Almheiri and J. Polchinski, J. High Energy Phys. 11 (2015) 014.

[5] K. Jensen, Phys. Rev. Lett. 117, 111601 (2016).

[6] J. Maldacena, D. Stanford, and Z. Yang, Prog. Theor. Exp. Phys. 2016, 12C104 (2016).

[7] J. Engelsöy, T. G. Mertens, and H. Verlinde, J. High Energy Phys. 07 (2016) 139.

[8] R. Jackiw, Nucl. Phys. B252, 343 (1985).

[9] C. Teitelboim, Phys. Lett. 126B, 41 (1983).

[10] J. S. Cotler, G. Gur-Ari, M. Hanada, J. Polchinski, P. Saad, S. H. Shenker, D. Stanford, A. Streicher, and M. Tezuka, J. High Energy Phys. 05 (2017) 118; 09 (2018) 002(E).

[11] A. M. García-García and J. J. M. Verbaarschot, Phys. Rev. D 94, 126010 (2016).

[12] P. Saad, S. H. Shenker, and D. Stanford, arXiv:1806.06840.

[13] P. Saad, S. H. Shenker, and D. Stanford, arXiv:1903.11115.

[14] E. Brezin and V. A. Kazakov, Phys. Lett. B 236, 144 (1990).

[15] M. R. Douglas and S. H. Shenker, Nucl. Phys. B335, 635 (1990).

[16] D. J. Gross and A. A. Migdal, Phys. Rev. Lett. 64, 127 (1990).

[17] D. J. Gross and A. A. Migdal, Nucl. Phys. B340, 333 (1990).

[18] G. 't Hooft, Nucl. Phys. B72, 461 (1974).

[19] E. Brezin, C. Itzykson, G. Parisi, and J. B. Zuber, Commun. Math. Phys. 59, 35 (1978).

[20] B. Eynard, J. High Energy Phys. 11 (2004) 031.

[21] M. Mirzakhani, Inventiones Mathematicae 167, 179 (2006).

[22] B. Eynard and N. Orantin, arXiv:0705.3600.

[23] B. Eynard and N. Orantin, Commun. Numer. Theor. Phys. 1, 347 (2007).

[24] T. R. Morris, Report No. FERMILAB-PUB-90-136-T, 1990.

[25] T. R. Morris, Nucl. Phys. B356, 703 (1991).

[26] S. Dalley, C. V. Johnson, and T. Morris, Nucl. Phys. B368, 625 (1992).

[27] S. Dalley, C. V. Johnson, and T. Morris, Nucl. Phys. B368, 655 (1992).

[28] S. Dalley, C. V. Johnson, and T. Morris, Nucl. Phys. B, Proc. Suppl. 25A, 87 (1992).

[29] I. R. Klebanov, J. Maldacena, and N. Seiberg, Commun. Math. Phys. 252, 275 (2004).
[30] K. Okuyama and K. Sakai, J. High Energy Phys. 01 (2020) 156.

[31] D. Stanford and E. Witten, arXiv:1907.03363.

[32] G. W. Moore, N. Seiberg, and M. Staudacher, Nucl. Phys. B362, 665 (1991).

[33] T. Banks, M. R. Douglas, N. Seiberg, and S. H. Shenker, Phys. Lett. B 238, 279 (1990).

[34] P. Ginsparg and G. W. Moore, arXiv:hep-th/9304011.

[35] C. A. Tracy and H. Widom, Commun. Math. Phys. 159, 151 (1994).

[36] A. M. García-García and S. Zacarías, arXiv:1911.10493.

[37] M. Pillai, J. Goglio, and T. G. Walker, Am. J. Phys. 80, 1017 (2012).

[38] J. E. Carlisle, C. V. Johnson, and J. S. Pennington, J. Phys. A 41, 085401 (2008).

[39] T. Nagao and K. Slevin, J. Math. Phys. (N.Y.) 34, 2075 (1993).

[40] C. A. Tracy and H. Widom, Commun. Math. Phys. 161, 289 (1994).

[41] A. Altland and M. R. Zirnbauer, Phys. Rev. B 55, 1142 (1997).

[42] S. Dalley, C. V. Johnson, T. R. Morris, and A. Watterstam, Mod. Phys. Lett. A 07, 2753 (1992).

[43] C. V. Johnson, arXiv:hep-th/0610223.

[44] S. P. Hastings and J. B. McLeod, Archive Rational Mech. Anal. 73, 31 (1980).

[45] J. E. Carlisle, C. V. Johnson, and J. S. Pennington, J. Phys. A 40, 12451 (2007).

[46] I. M. Gel'fand and L. A. Dikii, Russ. Math. Surv. 30, 77 (1975).

[47] R. Iyer, C. V. Johnson, and J. S. Pennington, J. Phys. A 44, 015403 (2011).

[48] R. Iyer, C. V. Johnson, and J. S. Pennington, J. Phys. A 44, 375401 (2011).

[49] T. R. Morris, Classical Quantum Gravity 9, 1873 (1992).

[50] C. V. Johnson, J. High Energy Phys. 12 (2004) 072.

[51] C. V. Johnson, arXiv:2005.01893.

[52] V. Periwal and D. Shevitz, Phys. Rev. Lett. 64, 1326 (1990).

[53] V. Periwal and D. Shevitz, Nucl. Phys. B344, 731 (1990).

[54] C. Crnkovic, M. R. Douglas, and G. W. Moore, Nucl. Phys. B360, 507 (1991).

[55] T. J. Hollowood, L. Miramontes, A. Pasquinucci, and C. Nappi, Nucl. Phys. B373, 247 (1992). 